\section{Residual Lattice Strains in Plastically Deformed Metals}

By measuring the change of the atomic interplanar spacing during a tensile (or compressive) test by means of X-ray diffraction methods, the 'lattice strain' can be plotted as a function of the stress. Such stress-v.-lattice strain curves, studied particularly by Smith and Wood ${ }^{1}$, show that tho lattice strain does not vanish when the applied stress is removed from the plastically deformed metal. This residual lattice strain must be due to microscopic residual stresses ${ }^{2}$, and an investigation of the residual strains helps to clarify the origin and distribution of these.

In the present communication it is shown that the internal stresses arise mainly from tho plastic anisotropy of the metal. The tensile stress required to cause glide in a given grain-the 'yield tension' of the grain-depends on the orientation of the glide planes and glide directions. In a polycrystalline aggregate deforming plastically under a tensile stress, grains with a high-yield tension will carry a greater stress than their neighbours, the orientations of which are such that they glide more easily. On removing the applied load a complicated stress system will arise in the aggregate; grains of high-yield tension will, in general, remain in tension, and those of low-yield tension will be in compression. The X-ray diffraction technique picks out grains of certain orientations, the normals to the reflecting planes being in a direction fixed geometrically by the X-ray beams. The 'residual lattice strain' found from a given $\mathrm{X}$-ray reflexion is not the mean of the strains of all the grains, which is zero, but a mean of the strains of grains with particular orientations. This in general will not be zero ; it must be expected to vary with the particular reflecting plano examined, and both positive and negative values should be found, depending on the choice of reflecting plane. On the other hand, an explanation of the residual lattice strain of the type proposed by Smith and Wood ${ }^{1}$ would seem to demand that all reflexions should show approximately the same value.

Experimental work carried out to test the validity of the hypothesis outlined was as follows. Annealed metal wires of about $0.5 \mathrm{~mm}$. diameter and extended plastically to the breaking point were photographed in a $19-\mathrm{cm}$. Bradley-Jay X-ray diffraction camera. Several characteristic X-radiations were used to obtain reflexions at high $\theta$ values for as many planes as possible. The strains for each reflecting plane were obtained by comparing the lattice spacings obtained from these photographs with those obtained from

RESIDUAL LATTICE STRAINS YOR VARIOUS REFLEOTING PLANES AFTER PLASTIC EXTENSION

\begin{tabular}{|c|c|c|}
\hline Metal & Plane reflecting & Residual lattice strain \\
\hline Iron & 310 & $+18 \times 10^{-5}$ \\
& 211 & +1 \\
110 & -31 \\
$10 \overline{1} 5$ & -3 \\
$12 \overline{3} 3$ & +3 \\
$11 \overline{2} 4$ & +2 \\
$20 \overline{2} 3$ & 0 \\
$10 \overline{1} 4$ & -7 \\
$20 \overline{2} 5$ & -3 \\
$12 \overline{3} 1$ & +4 \\
0006 & -5 \\
$30 \overline{3} 2$ & +2 \\
$20 \overline{2} 4$ & +3 \\
$30 \overline{3} 0$ & +4 \\
\hline
\end{tabular}

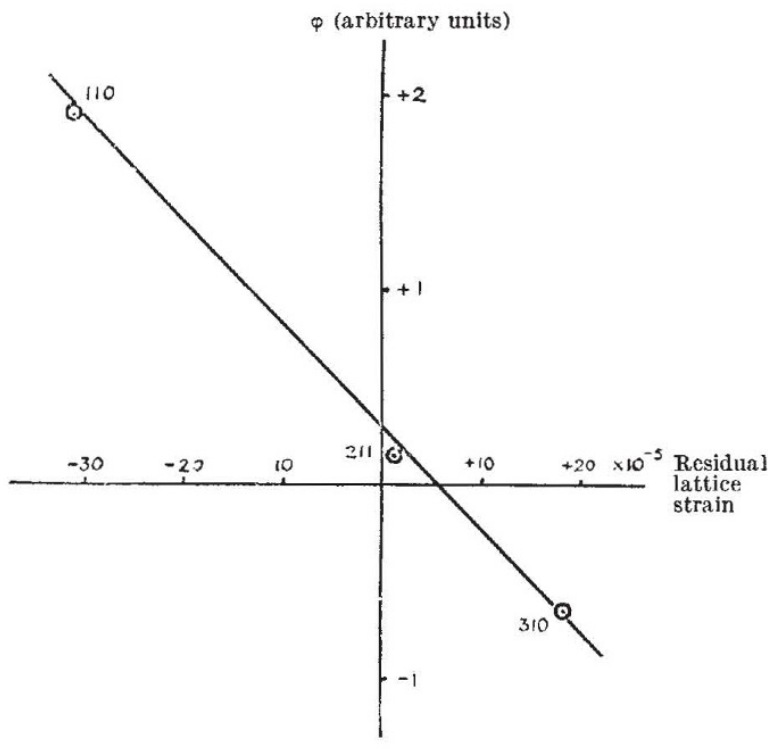

photographs taken of the same pieces of wire al ter re-annealing. The strains were always measured in a direction normal to that of the applied stress. In the results a residual lattice strain of sign opposite to the strain produced by the originally applied stress is denoted positive. Using this convention, the residual strains shown in the curves of Smith and Wood are always positive.

The table shows the results obtained for iron and for magnesium, the probable error of the residual lattice strains being about $1 \times 10^{-5}$.

For iron, a rough estimate of the average yield tension for grains of various orientations can be made from calculations performed for single crystals. If $\phi$ denotes the excess of the average yield tension for those grains contributing to a given reflexion over the mean value for all the grains in the aggregate then it can be shown that a plot of the residual lattice strain against $\varphi$ for various reflecting planes should be a straight line through the origin with a negative gradient. This graph is shown in the. figure.

Similar results have been obtained with copper and aluminium, and it is considered that they afford confirmation of the hypothesis put forward.

The calculations of the yield tension indicate that this quantity varies rapidly with the angle between the applied stress and the reflecting normal. This angle is $90^{\circ}$ in the experimental method used here, and about $80^{\circ}$ for the usual back-reflexion camera technique; it is impossible to compare results obtained by the two methods. Further work is being undertaken to investigate the possible effect of specimen size and shape on the residual lattice strain.

This work has been carried out for the Royal Aircraft Establishment as part of a programme for the development of the X-ray method of measuring locked-up stresses in fabricated metal parts.

\section{Cavendish Laboratory,} G. B. GREENOUSH Cambridge. May 12.

${ }^{1}$ Smith and Wood, Proc. Roy. Soc., A, 182, 404 (1944).

${ }^{2}$ Heyn, Festband K. W. Gesellschaft, 131 (1921). Masing, Wisc Siemens Konzern, 5, 135, 142 (1926). 